\title{
Comparative Study on CBIR based on Color Feature
}

\author{
Hany Fathy Atlam \\ Computer science and \\ Engineering Dept., Faculty of \\ Electronic Engineering, \\ Menoufia University, Menouf, \\ 32952, Egypt
}

\author{
Gamal Attiya \\ Computer science and \\ Engineering Dept., Faculty of \\ Electronic Engineering, \\ Menoufia University, Menouf, \\ 32952, Egypt
}

\author{
Nawal El-Fishawy \\ Computer science and \\ Engineering Dept., Faculty of \\ Electronic Engineering, \\ Menoufia University, Menouf, \\ 32952, Egypt
}

\begin{abstract}
Content Based Image Retrieval (CBIR) system helps users to retrieve relevant images based on their contents. It finds images in large databases by using a unique image feature such as texture, color, intensity or shape of the object inside an image. This paper presents a comparative study between the feature extraction techniques that based on color feature. These techniques include Color Histogram, HSV Color Histogram and Color Histogram Equalization. In this study, the retrieval process is first done by measuring the similarities between the query image and the images within the WANG database using two approaches: Euclidean distance and correlation coefficients. Then, the comparison is carried out by measuring the accuracy, error rate and elapsed time of each technique.
\end{abstract}

Keywords: CBIR, Feature Extraction, Image Retrieval, Similarity Matching, WANG database, Color Histogram.

\section{INTRODUCTION}

Impression is more by an image rather than thousands of words, as stipulated by the statement "a picture is worth a thousand words". Nowadays, a huge number of images are generated and transmitted in digital format over the Internet, and there is variety of tools to access the digital images. However, when searching for an image from the web using the Google image search, the retrieved results cannot meet the user satisfaction. This is because, the images searching by Google is based on associated metadata such as keywords, text, etc.

Recently, Content Based Image Retrieval (CBIR) techniques are developed to avoid the use of textual descriptions [1-5]. These techniques retrieve desired images from a large collection of image database. In CBIR, retrieving of images are based on some features in their contents like textures, colors or shapes which are the low level features of the image [6].

Figure 1 shows the block diagram of basic CBIR system. The CBIR involves two phases: feature extraction and feature matching. The first phase involves extracting the image features while the second phase involves matching these features. Briefly, the features of each image stored in the database is extracted and compared to the features of the query image. If the distance between feature vectors of the query image and the image in the database is small enough, the corresponding image in the database is to be considered as a matched image to the query. The matched images are then ranked accordingly to a similarity index. Finally, the retrieved images are specified according to the highest similarity.

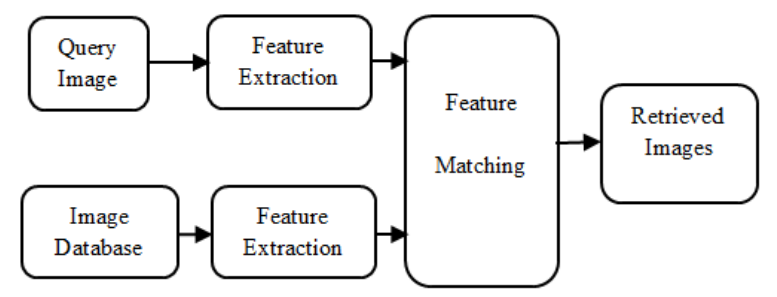

Fig 1: Block diagram of CBIR system

In this paper a comparative study is carried out on the CBIR techniques that based on color feature. The goal is to determine the most efficient feature extraction technique. In this study, the feature extraction process is done by using three algorithms: Color Histogram, HSV Color Histogram and Color Histogram Equalization. The retrieval process is first done by measuring the similarities between the query image and the images within the WANG database using two approaches: Euclidean distance and correlation coefficients. Then, the comparison is carried out by measuring the accuracy and error rate of each technique.

The rest of this paper is organized as follows. Section 2 presents an overview of related work. Section 3 briefly describes the Visual Feature Extraction techniques, section 4 briefly describes the Feature Matching Process, Section 5 discusses the experimental results, and finally Section 6 discusses the conclusion.

\section{REVIEW OF LITERATURE}

Content-based image retrieval, also known as query by image content or content-based visual information retrieval, is the application of computer vision to the image retrieval problem. That is, the problem of searching for digital images in large databases.

In the first generation, text annotations are used to retrieve the image [7]. However, Manual annotation is not always available and is impossible for a large database.To overcome the difficulties encountered by a text-based image retrieval system, 
content-based image retrieval was proposed in the early 1990s $[8,9]$.

The CBIR is used in many different fields, in Biomedicine fields such as X-ray, CT, medical diagnosis and security filtering, art galleries, museums, personal album etc. Some successful applications of the CBIR include:

- Texture segmentation and texture feature extraction.

- Fingerprints identification.

- Iris recognition.

- Edge detection.

- Directional image enhancement, image compression.

- Hierarchical image representation and recognition.

In Recent Years, several CBIR techniques have been done addressing different feature extraction techniques for image retrieval. In 2008, feature extraction techniques in CBIR using CT Brain image (gray scale image) were proposed in [10].

\section{FEATURE EXTRACTION TECHNIQUES}

In this section, the main concern is to describe the CBIR techniques that based on the color feature in image retrieval. The color feature has been widely used in the CBIR systems; because the color is an intuitive feature and plays an important role in image matching. In this paper, the feature extractions techniques that will be considered are: Color Histogram, HSV Color Histogram and Color Histogram Equalization.

\subsection{Color Histogram}

Color is one of the most important features in CBIR. It is the most widely used for both human perception \& computer vision. Image histogram is a graphical representation of an image. As we know image is a collection of pixels i.e. row and column, each pixel is associated to a specific histogram bin only on the basis of its own color, so the image histogram shows the proportion of pixels of each color within the image.

For the color histogram, the distribution of the number of pixels for each quantized bin can be defined for each component. Quantization in terms of color histograms refers to the process of reducing the number of bins by taking colors that are very similar to each other and putting them in the same bin.

The comparison between images (query image and images in the database) is accomplished through the use of some distance metric which determines the distance or similarity between the two histograms. The main advantage of histogram is variation in scale, rotation and translation of image $[11,12]$.

Figure 2 shows an example image for an elephant and its Histogram obtained by Color Histogram.
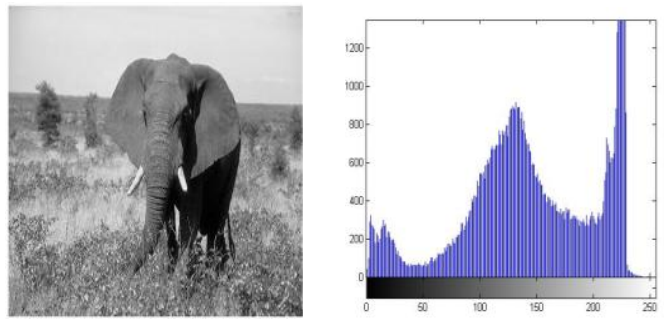

(a): an image

(b): Histogram of the image

Fig 2: elephant image and its Histogram

\subsection{HSV Color Histogram}

The representation of the colors in the RGB space is quite adapted for monitors, but for a human being, this is not a useful definition. To provide a user representation in the user interfaces, programmers prefer the HSV color space [13].

HSV Color Histogram is the acronym of Hue, Saturation and Value; this color histogram is the closest perception of the human eye. The human eye perceives colors by the excitation of two cells of the eye, which are rods and cones. "Excitation of the cone cells leads to perception of color, while rod cells help in perception of various shades of gray. The HSV color histogram separates the luminance component (Intensity) of a pixel color form its chrominance components (Hue and saturation)." This representation works as the human eye because it works like the separation of the rods and cones.

On the representation of the components for this histogram Hue represents the chromatic component; saturation represents the predominance value of a hue a color and Value represents the intensity of the color [14].

The generation of colors of the HSV color space is represented According to [15], Hue defines the color by changing its angle; hue is defined as an angle in the range $[0,2 \pi]$. Saturation is the depth or purity of the color and is measured as a radial distance from the central axis with value between 0 at the center to 1 at the outer space. Finally, value is represented by the vertical central axis. Figure 3 illustrate how HSV color histogram is represented.

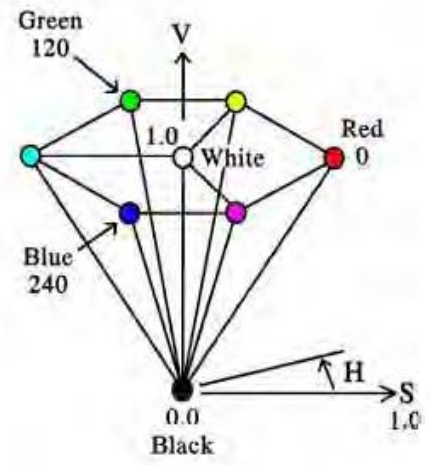

Fig 3: Representation of a HSV color descriptor. 


\subsection{Color Histogram Equalization}

The Histogram Equalization spreads out intensity values along the total range of values in order to achieve higher contrast. This method is especially useful when an image is represented by close contrast values, such as images in which both the background and foreground are bright at the same time, or else both are dark at the same time [16].

For example, the result of applying histogram equalization to the image in Figure 4 is presented in Figure 5. We can see that the image's contrast has been improved. The original histogram has been stretched along the full range of gray values.

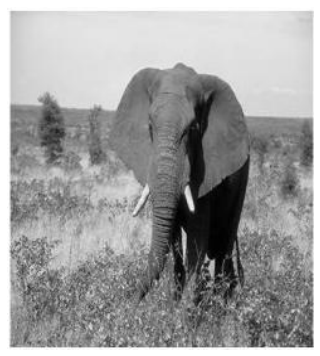

(a): an image

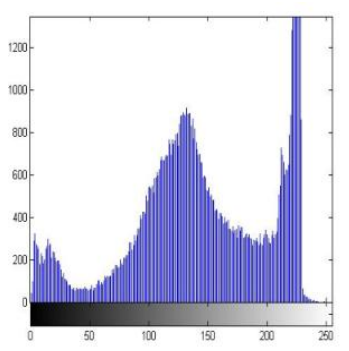

(b): the image Histogram Fig 4: elephant image and its Histogram

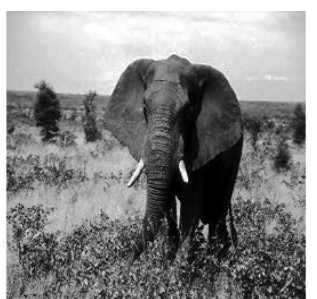

(a): an image

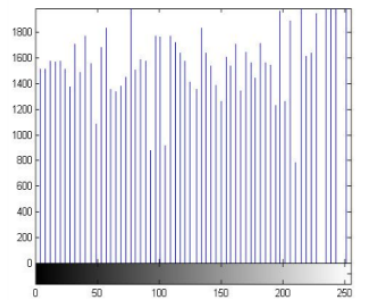

(b): the Histogram Equalization

\section{FEATURE MATCHING PROCESS}

The Similarity matching is the process of approximating a solution, based on the computation of a similarity function between a pair of images.

To retrieve the similar images from the large image database, two types of similarity matching algorithms like Histogram Euclidean distance and correlation coefficients are being used.

\subsection{Histogram Euclidean distance}

Let $\mathrm{h}$ and $\mathrm{g}$ represent two color histograms. The Euclidean distance between the color histograms $\mathrm{h}$ and $\mathrm{g}$ can be computed as [17]:

\subsection{Correlation Coefficient $(\mathrm{Cr})$}

The closeness between the original image and the modified one is measured by the correlation coefficient $(\mathrm{Cr})$. In ideal case the $\mathrm{Cr}$ should be one.

$$
\mathrm{C} r=\frac{\sum_{i=\mathbf{1}}^{n}\left(X_{i}-\bar{X}\right)\left(Y_{i}-\bar{Y}\right)}{\sqrt{\sum_{i=1}^{n}\left(X_{i}-\bar{X}\right)^{2}} \sqrt{\sum_{i=1}^{n}\left(Y_{i}-\bar{Y}\right)^{2}}}
$$

Where: $\mathrm{X}$ is the query image, $\mathrm{Y}$ is the image from the database and $n$ is the number of images in the database.

\section{EXPERIMENTAL STUDY}

\subsection{Simulation Environment}

The feature extraction algorithms and similarity matching methods are coded using MATLAB [18] on Intel(R) Core(TM) i5 CPU with 4GB RAM running Windows 7 and several experiments are carried out to evaluate the performance of the system.

In our experiments, The WANG database which is a standard database is being used to evaluate the performance of the system. The WANG database is a subset of the Corel database of 1000 images which have been manually selected to be a database of 10 categories of 100 images each. The images are of the size $256 \times 384$.

The images are subdivided into 10 categories (African People, Beach, Buildings, Buses, Dinosaurs, Elephants, Flowers, Horses, Mountains and Foods) such that it is almost sure that a user wants to find the other images from a category if the query is from one of these 10 categories. This is a major advantage of this database because due to the given classification it is possible to evaluate retrieval results.

One example of each category can be seen in Figure 6. This database was used extensively to test the different features because the size of the database and the availability of class information allows for performance evaluation [19, 20].

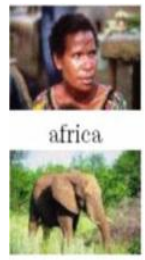

elephants

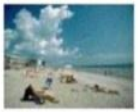

beach

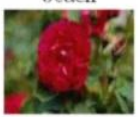

flowers

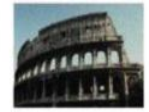

monuments

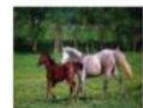

Horses

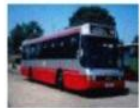

buses

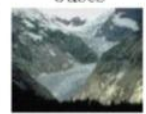

Mountains

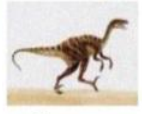

dinosaurs

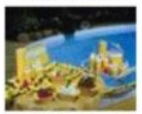

Foods
Fig 6: One example image from each of the 10 categories of the WANG database

$$
\mathrm{D}=\sqrt{\sum(\mathrm{h}-\mathrm{g})^{2}}
$$




\subsection{Performance Evaluation}

The performance of the system can be evaluated by using a set of parameters such as $[21,22]$ :

\subsubsection{Time}

It is the time taken in seconds for the retrieval task to complete, at the end of which the system returns the images which are matched with the features of the query images, according to the technique used.

\subsubsection{Accuracy}

Accuracy of an image retrieval task is defined as the ratio of the number of relevant images retrieved to the total number of images retrieved expressed in percentage.

\subsubsection{Error rate}

Error rate of an image retrieval task is defined as the ratio of the number of irrelevant images retrieved to the total number of images retrieved expressed in percentage.

\subsection{Results}

To evaluate the performance of each Feature Extraction algorithm the following steps will be carried out:

1. Select five images from each category as a query images.

2. For each retrieval, the most similar 20 images are selected as retrieval results

3. Compute the accuracy, the error rate and the elapsed time for the 5 query images.

4. Compute the average accuracy, the average error rate and the average execution time of the 5 retrievals.

Table 1 shows the average retrieval accuracy and the average retrieval time that result from applying the three feature extraction algorithms on the WANG database using the Euclidean distance as a Feature similarity measure.

The experimental results show that the HSV color histogram and color histogram algorithm gives the best retrieval accuracy but with different execution time.
Table 1: Comparison between the three Feature Extraction algorithms

\begin{tabular}{|c|c|c|c|c|c|c|}
\hline Algorithm & & $\begin{array}{l}\text { olor } \\
\text { ogram }\end{array}$ & & $\begin{array}{l}\text { Color } \\
\text { gram }\end{array}$ & & $\begin{array}{l}\text { olor } \\
\text { ogram } \\
\text { lization }\end{array}$ \\
\hline $\begin{array}{l}\text { Image } \\
\text { type }\end{array}$ & $\begin{array}{l}\text { Acc } \\
(\%)\end{array}$ & $\begin{array}{l}\text { Time } \\
\text { (sec) }\end{array}$ & $\begin{array}{l}A c c \\
(\%)\end{array}$ & $\begin{array}{l}\text { Time } \\
\text { (sec) }\end{array}$ & $\begin{array}{l}\text { Acc } \\
(\%)\end{array}$ & $\begin{array}{l}\text { Time } \\
(\mathrm{sec})\end{array}$ \\
\hline African & 80 & 10.929 & 96 & 62.277 & 25 & 31.030 \\
\hline Beach & 25 & 10.071 & 60 & 63.598 & 21 & 29.755 \\
\hline Buildings & 31 & 10.957 & 57 & 60.416 & 21 & 28.457 \\
\hline Buses & 46 & 10.689 & 83 & 60.690 & 37 & 27.952 \\
\hline Dinosaurs & 98 & 10.721 & 100 & 58.934 & 50 & 28.367 \\
\hline Elephants & 39 & 10.364 & 47 & 71.741 & 35 & 30.741 \\
\hline Flowers & 91 & 10.286 & 100 & 61.726 & 43 & 29.027 \\
\hline Horses & 51 & 10.736 & 94 & 61.586 & 18 & 28.823 \\
\hline $\begin{array}{l}\text { Mountain } \\
\text { S }\end{array}$ & 25 & 13.572 & 25 & 61.258 & 20 & 28.595 \\
\hline Foods & 42 & 13.962 & 85 & 62.206 & 25 & 28.151 \\
\hline
\end{tabular}

Figure 7 shows an example of the retrieval result using the Color Histogram Algorithm and the query image belongs to the buses category from the Wang database.
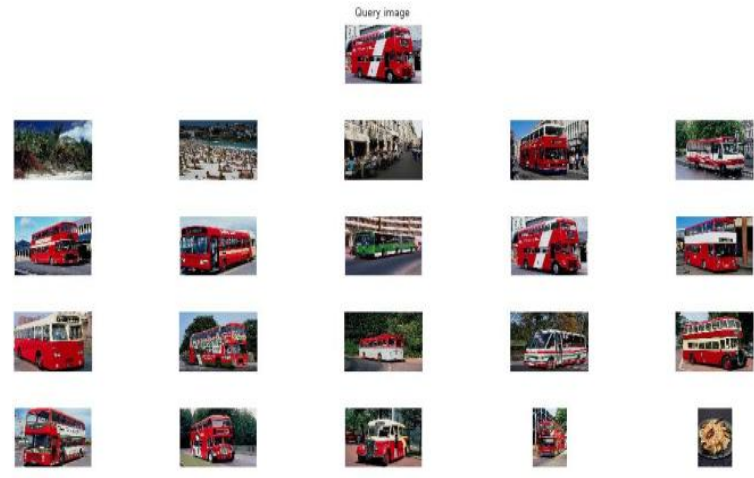

Fig 7: Example of Retrieval of images using Color Histogram 
Figure 8 shows an example of the retrieval result using the HSV Color Histogram Algorithm and the query image belongs to the Dinosaurs category from the Wang database.

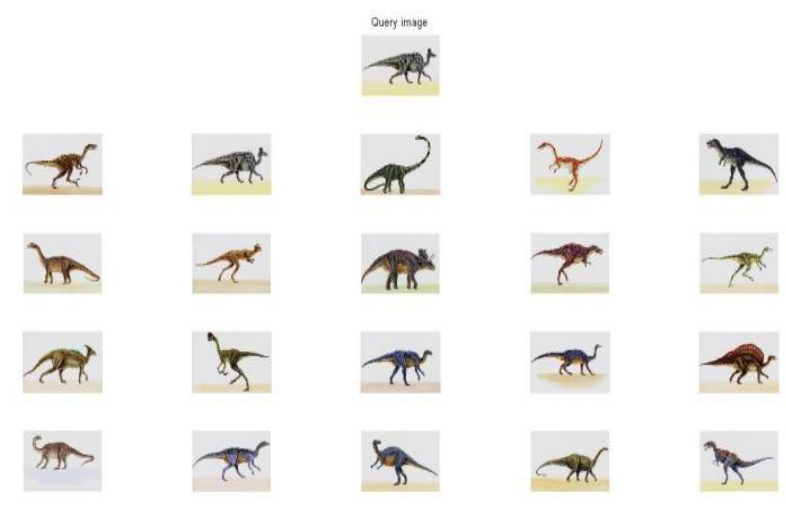

Fig 8: Example of Retrieval of images using HSV Color Histogram

Figure 9 shows an example of the retrieval result using the Color Histogram Equalization Algorithm and the query image belongs to the Elephants category from the Wang database.

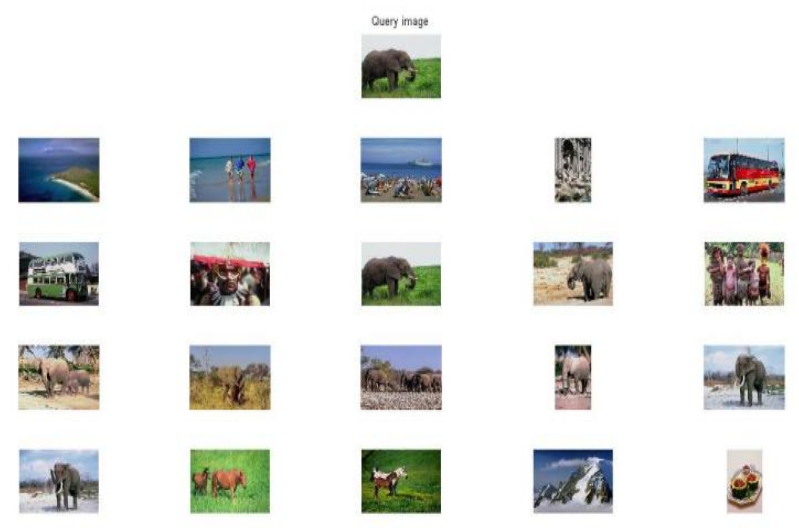

Fig 9: Example of Retrieval of images using Color Histogram Equalization

Table 2 shows the average retrieval accuracy and average retrieval time that results from applying the three feature extraction algorithms on the WANG database and using a correlation ratio $(\mathrm{Cr}=0.85)$ as a Feature similarity measure.
Table 2: Comparison between the three Feature Extraction algorithms

\begin{tabular}{|c|c|c|c|c|c|c|}
\hline Algorithm & \multicolumn{2}{|c|}{$\begin{array}{c}\text { Color } \\
\text { Histogram }\end{array}$} & \multicolumn{2}{|c|}{$\begin{array}{c}\text { HSV Color } \\
\text { Histogram }\end{array}$} & \multicolumn{2}{c|}{$\begin{array}{c}\text { Color } \\
\text { Histogram } \\
\text { Equalization }\end{array}$} \\
\hline $\begin{array}{c}\text { Image } \\
\text { type }\end{array}$ & $\begin{array}{c}\text { Acc } \\
(\%)\end{array}$ & $\begin{array}{c}\text { Time } \\
(\text { Sec })\end{array}$ & $\begin{array}{c}\text { Ac } \\
\boldsymbol{c} \\
(\%)\end{array}$ & $\begin{array}{c}\text { Time } \\
(\text { Sec })\end{array}$ & $\begin{array}{c}\text { Acc } \\
(\%)\end{array}$ & $\begin{array}{c}\text { Time } \\
(\text { Sec })\end{array}$ \\
\hline African & 76 & 5.581 & 91 & 36.269 & 25 & 14.228 \\
\hline Beach & 45 & 5.475 & 73 & 30.404 & 19 & 13.948 \\
\hline Buildings & 63 & 5.360 & 57 & 29.191 & 23 & 14.320 \\
\hline Buses & 75 & 5.079 & 80 & 29.078 & 38 & 15.235 \\
\hline Dinosaurs & 100 & 6.683 & 100 & 30.904 & 38 & 13.182 \\
\hline Elephants & 75 & 5.416 & 70 & 30.841 & 34 & 14.238 \\
\hline Flowers & 95 & 5.397 & 82 & 29.359 & 41 & 13.256 \\
\hline Horses & 68 & 5.441 & 97 & 29.437 & 21 & 13.257 \\
\hline Mountain \\
s
\end{tabular}

The experimental results show HSV Color Histogram algorithm gives the best retrieval accuracy.

Figure 10 shows an example of the retrieval result using the Color Histogram Algorithm and the query image belongs to the Flowers category from the Wang database.

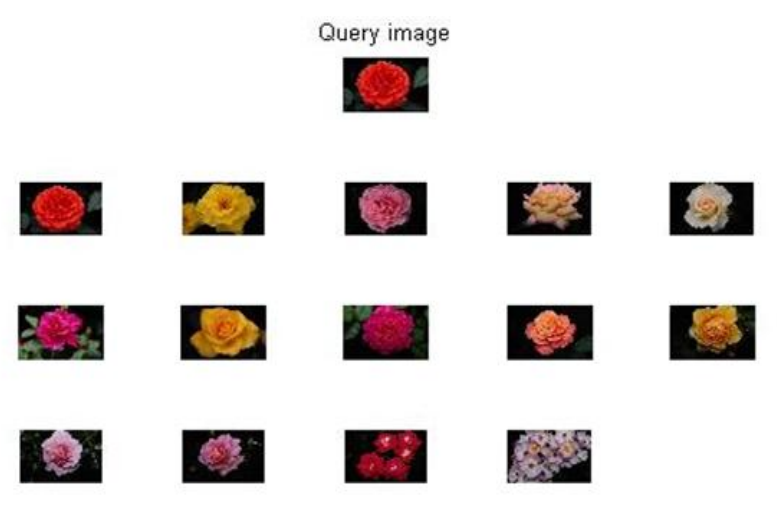

Fig 10: Example of Retrieval of images using Color Histogram

Figure 11 shows an example of the retrieval result using the HSV Color Histogram Algorithm and the query image belongs to the African category from the Wang database. 


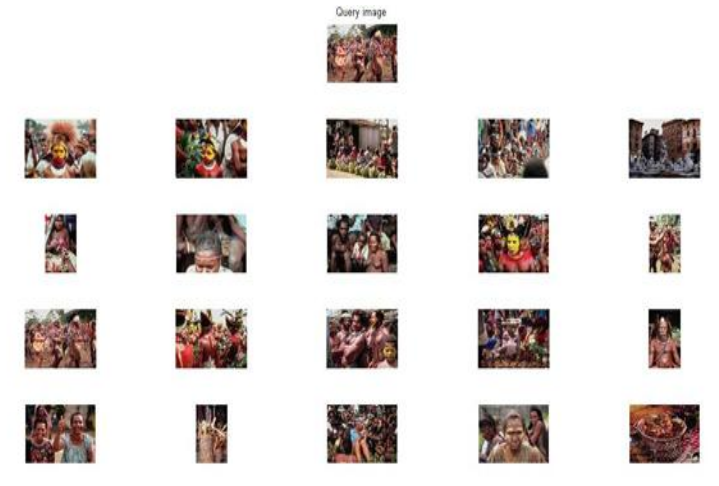

Fig 11: Example of Retrieval of images using HSV Color Histogram

Figure 12 shows an example of the retrieval result using the Color Histogram Equalization Algorithm and the query image belongs to the Food category from the Wang database.

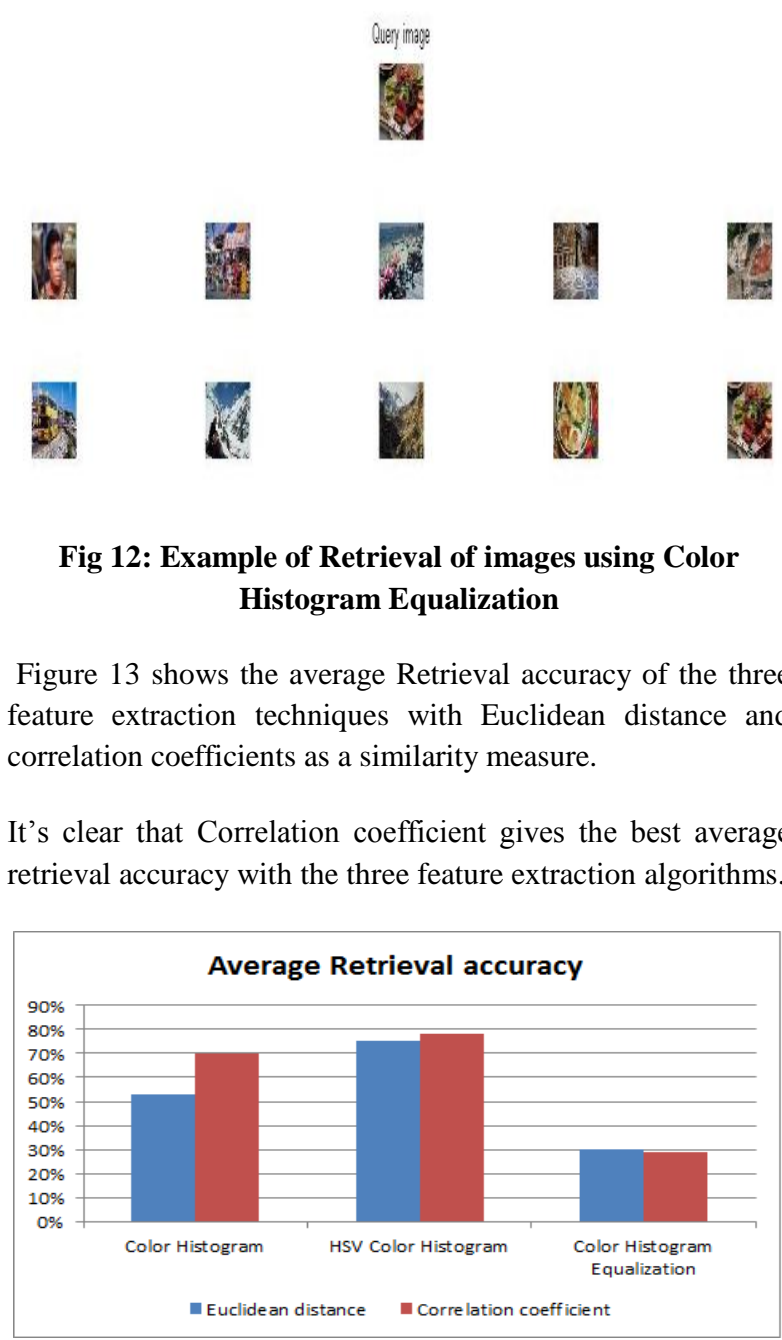

Fig 13: average Retrieval Accuracy with similarity measure
Figure 14 shows the average Retrieval time of the three feature extraction techniques with Euclidean distance and correlation coefficients as a similarity measure.

Although the Color Histogram algorithm gives the best retrieval time with both Euclidean distance and correlation coefficient but the retrieval accuracy is low.

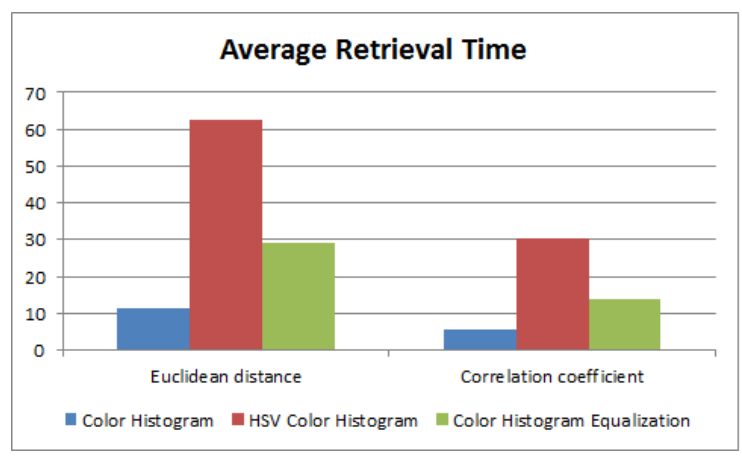

Fig 14: average Retrieval time with similarity measure

\section{CONCLUSION}

In this paper, a comparative study had been carried out on three feature extraction techniques that based on color feature. Each technique is applied to retrieve images from the WANG database. The comparison is done by measuring the accuracy, the error rate and the elapsed time. The Experimental results show that the HSV Color Histogram is the most efficient feature extraction technique. Indeed, the correlation coefficient is the best feature similarity measure method.

\section{REFERENCES}

[1] D. Feng, W. C. Siu, and H. J. Zhang, "Fundamentals of Content-Based Image Retrieval", Multimedia Information Retrieval and Management-Technological Fundamentals and Applications,New York:Springer ,2003.

[2] V. N. Gudivada and V. V. Raghavan, "Content based image retrieval systems", IEEE Computer, vol. 28, Sept. 1995.

[3] G. Pass and R. Zabih, "Histogram refinement for contentbased image retrieval," in 3rd IEEE Workshop on Applications of Computer Vision, pp. 96-102,1996.

[4] L. Cinque, S. Levialdi, and A. Pellicano, "Color-based image retrieval using spatial-chromatic histograms," in IEEE Int. Conf. Multimedia Computing and Systems, pp. 969-973,1999.

[5] D. Ashok Kumar and J. Esther, "Comparative Study on CBIR based by Color Histogram Gabor and Wavelet Transform", International Journal of Computer Applications, Vol. 17, No. 3, March 2011.

[6] N. S. Chang and K.S.Fu, "Image Query by Pictorial Example,” IEEE Trans. Software Engineering, 1980.

[7] Y. Rui and T.S. Huang, "Image Retrieval: Current Techniques, Promising Directions, and Open issues, 
Visual Commun, Image Representation”, pp. 39-62, 1999.

[8] A.W.M. Smeulders, "Content-based image retrieval at the end of the early years", IEEE Trans. Pattern Anal. Mach. Intel, Vol. 22, pp. 1349-1379, 2000.

[9] Wan Siti Halimatul Munirah Wan Ahmad and Mohammad Faizal Ahmad Fauzi, "Comparison of Different Feature Extraction Techniques in ContentBased Image Retrieval for CT Brain images", Multimedia Signal Processing, IEEE 10th Workshop on, pp. $503-508,2008$.

[10] Manimala Singha and K.Hemachandran, "Content Based Image Retrieval using Color and Texture", Signal \& Image Processing: An International Journal (SIPIJ), Vol. 3, No. 1, February 2012.

[11] D.A. Kumar and J. Esther, "Comparative Study on CBIR based by Color Histogram, Gabor and Wavelet Transform”, Vol. 17, No.3, pp. 37-44, March 2011.

[12] R. Chakarvarti and X. Meng, "A Study of Color Histogram Based Image Retrieval", Information Technology: New Generations, ITNG '09. Sixth International Conference on, pp. 1323-1328,2009.

[13] Youngeun an and Muhammad Riaz and Jongan Park, "CBIR based on adaptive segmentation of HSV color space" 12th International Conference on Computer Modelling and Simulation, 2010.

[14] J. Miralles, Tutorial de GIMP. [Online]. Available: http: //sites.google.com/site/tutorialdegimp/011---teoria-delcolor-for\%macion-y-mezcla-de-colores -rgb y-cmyk.
[15] A. Vadivel, S. Sural, and A. Majumdar, "Human color perception in the HSV space and its aplication in histogram generation for image retrieval," in SPIE Procedings seetings, San José CA,United States of America, 2005.

[16] P.B Thawari,N.J janwe,"CBIR Based on Color and Texture", International Journal of Information Technology and Knowledge Management,Vol.4,No.1,pp. 129-132,june 2011

[17] Liwei Wang, Yan Zhang, and Jufu Feng. "On the Euclidean Distance of Images", IEEE TRANSACTIONS ON PATTERN ANALYSIS AND MACHINE INTELLIGENCE, VOL. 27, NO. 8, AUGUST 2005.

[18] MATLAB - the language of technical computing, http://www.mathworks.com/products/matlab/index.html

[19] J. Li, J.Z. Wang, "Automatic linguistic indexing of pictures by a statistical modeling approach", IEEE Transactions on Pattern Analysis and Machine Intelligence, Vol. 25, No. 9, pp. 1075-1088, 2003.

[20] Wang's dataset http://wang.ist.psll.edll/

[21] Aman_Chadha,Sushmit Mallik and Ravdeep Johar," Comparative Study and Optimization of Feature Extraction Techniques for Content based Image Retrieval", International Journal of Computer Applications Vol. 52- No.20, August 2012.

[22] S. Manimala and K. Hemachandran, "Image RetrievalBased on Color Histogram and performance Evaluation of similarity Measurement", Assam University Journal of science \& Technology, Vol. 8 Number II ,pp.94-104, 2011. 